\title{
Adult Colo-Colic Intussusception: Lipoma or Cancer??
}

Zhar Jamal", Bahri Mohamed Oussama, Anis Tarek, Ouadii Mouaqit, El Bouhaddouti Hicham, El Benjelloun Bachir, Abdelmalek Ousadden, Ait Taleb Khalid

Department of General Surgery A, CHU Hassan II FES, 30000, Morocco

DOI: $10.36347 /$ sjmcr.2020.v08i04.005

| Received: 05.03.2020 | Accepted: 19.03.2020 | Published: 05.04.2020

*Corresponding author: Jamal Zhar

Abstract

Case Report

Acute intestinal intussusception in adults is rare [1]. In majority of adult cases, there is an underlying cause such as polyps or colon cancers. Invaginations of the small intestine account for $75 \%$ and $25 \%$ of colonic origin. The diagnosis is difficult and often late [2]. We report a rare case of acute intestinal intussusception on the polyp of the transverse colon on a 65-year-old woman, whose diagnosis was made using an abdominopelvic CT scan. Through this observation and a review of the literature we will try to identify the clinical and therapeutic features of this entity.

Keywords: Intussusception, adult, cancer, lipoma, colectomy.

Copyright @ 2020: This is an open-access article distributed under the terms of the Creative Commons Attribution license which permits unrestricted use, distribution, and reproduction in any medium for non-commercial use (NonCommercial, or CC-BY-NC) provided the original author and source are credited.

\section{CASE}

A 65 old woman with a history of inflammatory rheumatism for 03 years on corticosteroid therapy. Who presented rectal bleeding 1 year ago with intermittent and colicky abdominal pain, she had a generalcachexia.The physical exam a sensitivity of the left hypochondrium and Normal rectal examination.

Colonoscopy: Presence at the splenic flexure, a large sessile $4 \mathrm{~cm}$ polyp, classified $\mathrm{Vi}$ according to the Kudo classification (figure 1). Pathological examination revealeda villous tubular adenoma.

An endoscopic polypectomy was unsuccessful. Computed tomography (CT) showed bowel-withinbowel appearance of colo-colic intussusception elongated from the transverse to the left colon (figure2).

An exploratory laparotomy confirmed the diagnosis as well as the aetiology of an intraluminalpolyp. Left hemicolectomy with primary anastomosis was performed (figure3.4). Pathological examination revealed colonic adenocarcinoma.

After an unremarkable postoperative course, she was doing well at the 3-month follow-up.

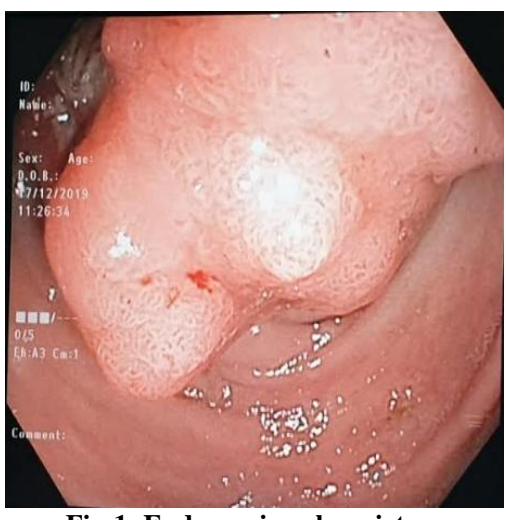

Fig-1: Endoscopic polyp picture

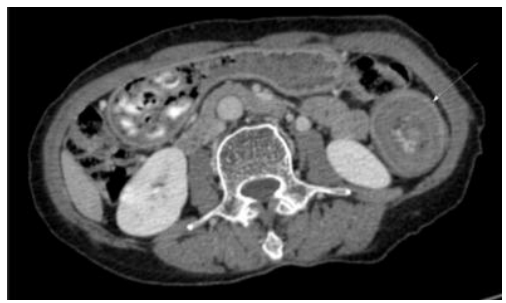

Fig-2: CT scan picture of intussusception

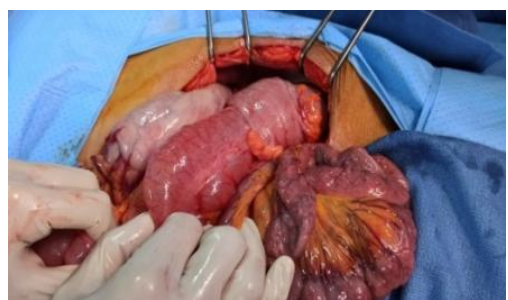

Fig-3: Per operative picture of intussusception 


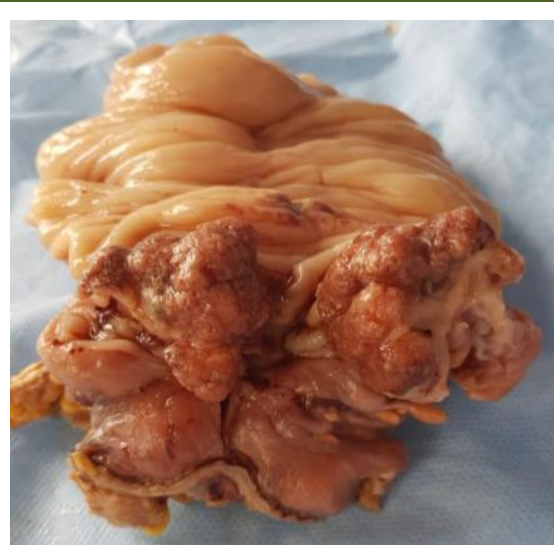

Fig-4: Macroscopic pathological examination

\section{Discussion}

Acute intussusception is rare in adults. It is rarely pure colic [2]. Distal colic forms represent only 2.1 to $9.4 \%$. It is often secondary to an organic lesion is this in $85 \%$ in data [1]. A malignant tumor has been reported as the common cause of colo-colonic intussusception in adults; however, benign tumors including lipomas are the main causes of enteroenteric ones. The polyp can be found at all levels of the digestive tract, but it is most commonly found in the colon $(70 \%)[3]$.

The clinical presentation is nonspecific, ranging from atypical abdominal pain to occlusion.The physical examination is based on abdominal palpation and digital rectal examination, which objectify the extension of intussusceptionin 24 to $42 \%$, but this frequency is depending on the series [4].

The radiological diagnosis can be made by the abdominal X-ray, but mainly by ultrasound and abdominal CT scan. The ultrasound shows the classic target sign with a double digestive wall, but also ischemic necrosis by Doppler [5].

The Computed tomography is a a sensitive and specific tool for the diagnostis, itconfirme the bowel intusseception, its location and the nature of the etiology, also it appreciate the degree of visceral suffering.

In adults, the treatment of an intussusception is always surgical [8]. Because of the frequency of cancer as a causal lesion in colo-colic or ileo-colic invaginations, the first resection (regulated colectomy) is recommended. While in small intussusception, a reduction prior to limiting the extent of the excision is preferred given the rarity of malignant tumors $[9,10]$.

\section{Conclusion}

Although it is a very rare event in adult population, intussusception can be seen in the presence of an underlying pathology including a polyp or malignancy. Enteroenteric small intestinal intussusception is the most common type in adults; however, ileo-colo-colonic intussusception with anal protrusion may occur in the absence of an organic cause. Bowel resection en-bloc without reduction should be offered as the management strategy in adults due to the suspicious malignant causes.

\section{REFFERENCE}

1. Oyen TL, Wolthuis AM, Tollens T, Aelvoet C, Vanrijkel JP. Ileo-ileal intussusception secondary to a lipoma: a literature review. Acta chirurgica Belgica. 2007 Jan 1;107(1):60-3.

2. Oukachbi N, Brouzes S. Invagination intestinale de l'adulte due à un lipome de l'intestin grêle. Gastroentérologie clinique et biologique. 2010;34(6-7):413-5.

3. Abid M, Mzali R, Feriani N, Guirat A, Boujelbene S, Frikha M, Amar M, Beyrouti M. Un lipome colique à l'origine d'une invagination intestinale aiguë chez l'adulte à propos d'un cas. Journal de l'Information Médicale de Sfax. 2009:32.

4. Guillén-Paredes MP, Campillo-Soto A, MartínLorenzo JG, Torralba-Martínez JA, MengualBallester M, Cases-Baldó MJ, Aguayo-Albasini JL. Adult intussusception-14 case reports and their outcomes. Revista Espanola De Enfermedades Digestivas. 2010 Jan 1;102(1):32.

5. Nylund K, degaard S, Hausken T, Folvik G, Lied GA, Viola I, Hauser H, Gilja OH. Sonography of the small intestine. World J Gastroenterol. 2009;15:1319-1330.

6. Beattie GC, Peters RT, Guy S, Mendelson RM. Computed tomography in the assessment of suspected large bowel obstruction. ANZ J Surg. 2007 Mar;77(3):160-5.

7. Sandrasegaran K, Kopecky KK, Rajesh A, Lappas J. Proximal small bowel intussusceptions in adults: CT appearance and clinical significance. Abdom Imaging. 2004 Nov-Dec;29(6):653-7.

8. Sebbag H, Brunaud L, Marchal F, Collinet-Adler S, Grosdidier G. Intestinal intussusception in adults, treat it like a cancer. Oncol Rep. 2000 NovDec;7(6):1359-61.

9. Tsushimi T, Matsui N, Kurazumi H, Takemoto Y, Oka K, Seyama A, Morita T. Laparoscopic resection of an ileal lipoma: report of a case. Surgery today. 2006 Nov 1;36(11):1007-11.

10. Kunduz E, Malya FÜ, Mehdi E, Hasbahçeci M. A rare cause of obstructive defecation in a 29-yearold woman: Ileo-colo-colonic intussusception treated by subtotal colectomy with posterior rectopexy. Turkish journal of surgery. 2018;34(4):334. 